\title{
Thermal Inelastic Collision Processes
}

\author{
M. J. Seaton \\ Department of Physics, University College, University of London, London, England
}

\section{OUTLINE}

$S^{\mathrm{E}}$ ECTION II contains a summary of relevant collision theory methods. Section III is concerned with heavy particle collisions: excitation of $\mathrm{H} 1 s$ hyperfine structure (hfs) states by $\mathrm{H}$ atom impact; excitation of $\mathrm{H}_{2}$ rotation by $\mathrm{H}$ and $\mathrm{H}_{2}$ impact; $\mathrm{H} 2 s \rightarrow 2 p$ transitions produced by proton impact; charge exchange reactions; excitation of atomic levels by proton impact. Section IV deals with inelastic collisions between atoms and electrons. Use of the Born and distorted wave approximations is discussed. Calculations of cross sections for excitation of forbidden lines in $p^{q}$ configurations are reviewed and new results presented for $\mathrm{O}^{+2}, \mathrm{~N}^{+}, \mathrm{C}^{+}$, and $\mathrm{Si}^{+}$, both variational and semiempirical methods being used. In Sec. V, concerned with atomic photoionization, new results are given for photoionization from $2 p^{q}$ configurations.

\section{GENERAL THEORY}

\section{Cross Sections and Reaction Rates}

We consider reactions of the type

$$
A_{i}+B \rightarrow A_{j}+B,
$$

where $A_{i}, A_{j}$ denote particle $A$ in state $i$ or state $j$. With particle densities $n\left(A_{i}\right), n(B)$ and initial relative velocity $v_{i}$ the rate of collsions of type (1) is $n\left(A_{i}\right) n(B)$ $\times q_{i j}$, where $q_{i j}=v_{i} Q(i j)$ and $Q(i j)$ is the effective cross section. With a normalized velocity distribution function $f(v)$ the rate coefficient becomes

$$
q_{i j}=\int v_{i} Q(i j) f\left(v_{i}\right) d v_{i} .
$$

Let $E_{i}$ be the internal energy of $A_{i}, E_{j}$ that of $A_{j}$. In thermodynamic equilibrium the Boltzmann relation

$$
\frac{n\left(A_{i}\right)}{n\left(A_{j}\right)}=\frac{\omega_{i}}{\omega_{j}} \exp \left[\left(E_{j}-E_{i}\right) / k T\right]
$$

( $\omega_{i}, \omega_{j}$ being statistical weights) combined with the detailed balance relation

$$
n\left(A_{i}\right) n(B) q_{i j}=n\left(A_{j}\right) n(B) q_{j i}
$$

gives the relation

$$
q_{i j}=\left(\omega_{j} / \omega_{i}\right) q_{j i} \exp \left[-\left(E_{j}-E_{i}\right) / k T\right] .
$$

On substituting a Maxwell distribution in (2) and requiring (5) to be true for all $T$, we obtain

$$
\omega_{i} v_{i}^{2} Q(i j)=\omega_{j} v_{j}^{2} Q(j i) \text {. }
$$

A mean cross section, averaged over a Maxwell distribution, is often defined by

$$
q=\bar{v} \bar{Q}
$$

where $\bar{v}=(8 k T / \pi M)^{\frac{1}{1}}$ is the mean thermal velocity. Cross sections are frequently expressed in units of $a_{0}^{2}=2.803 \times 10^{-17} \mathrm{~cm}^{2}$ or $\pi a_{0}^{2}=8.806 \times 10^{-17} \mathrm{~cm}^{2}$.

\section{Classical Treatment}

In classical theory definite particle trajectories must be considered, each trajectory being characterized by an impact parameter $R$ (Fig. 1). With $R$ in the range $d R$ let $P(i, j ; R)$ be the probability of the reaction (1) occurring during collision. The total collision rate with $R$ in $d R$ is $n\left(A_{i}\right) n(B) v_{i} \cdot 2 \pi R d R$ and the cross section for (1) is therefore

$$
Q(i j)=\int_{0}^{\infty} P(i, j ; R) 2 \pi R d R .
$$

Clearly,

$$
P(i, j ; R) \leq 1 \text {. }
$$

For the calculation of $P$, various mechanisms may be considered. If $B$ is a charged particle, it will produce a variable field $\mathbf{E}(R, t)$ in the neighborhood of $A$. We make a Fourier analysis of this field, pick out the component $E_{\nu}$ (where $h \nu=\left|E_{j}-E_{i}\right|$ ), and calculate $P$ as the probability of an induced radiative transition. The calculated values of $P$ must be made consistent with (8). This method requires the kinetic energy of $B$ to be large compared to the transition energy $\left(E_{j}-E_{i}\right)$, since no allowance is made for a change of kinetic energy when the transition occurs. If it is also assumed that $B$ is not appreciably deflected during collision, it may be expected that $Q$ will depend on the velocity of $B$ but not on its mass.

For atom-electron collisions the collision mechanism may involve capture of the incident electron and ejection of an atomic electron. From a classical standpoint this would represent a distinct physical process.

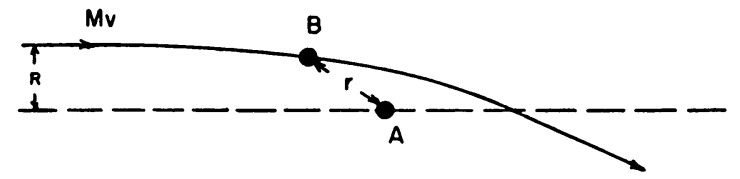

Fig. 1. The impact parameter $R$ would be equal to the distance of closest approach if the colliding particles were undeflected. 


\section{Quantal Treatment}

Quantization of angular momentum results from putting $L=M v R$ and $L^{2}=\hbar^{2} l(l+1)$, with $l$ integral. Then with $R_{l}=[l(l+1)]^{\frac{1}{3}} / k_{i}, k_{i}=M v_{i} / \hbar$, and $\delta l=1$, we have

$$
2 R \delta R=(2 l+1) / k_{i}{ }^{2} .
$$

Replace the integral (7) by a sum,

$$
Q(i j)=\frac{\pi}{k_{i}{ }^{2}} \sum_{l=0}^{\infty}(2 l+1) P\left(i, j ; R_{l}\right) .
$$

We now put $\Omega^{l}(i, j)=(2 l+1) P\left(i, j ; R_{l}\right)$ and

giving

$$
\Omega(i, j)=\sum_{l} \Omega^{l}(i, j)
$$

$$
Q(i j)=\left(\pi / k_{i}{ }^{2}\right) \Omega(i, j)
$$

The dimensionless parameter ${ }^{1} \Omega(i, j)$ is the collision strength. The reciprocity relation (6) gives $\Omega(i, j)=\Omega(j, i)$ and the conservation relation (8) gives

$$
\Omega^{l}(i, j) \leq(2 l+1) .
$$

With degenerate states $i_{s}\left(s=1,2, \cdots \omega_{i}\right)$ and $j_{t}$ $\left(t=1,2, \cdots \omega_{j}\right)$ the cross section is

with

$$
Q(i j)=\frac{\pi}{k_{i}{ }^{2} \omega_{i}} \Omega(i, j)
$$

$$
\Omega(i, j)=\sum_{s, t} \Omega\left(i_{s}, j_{t}\right) .
$$

At temperature $T\left({ }^{\circ} \mathrm{K}\right)$ the rate coefficient for deactivation, $j \rightarrow i$ with $E_{j}>E_{i}$, is

$$
\begin{aligned}
q_{j i}=\frac{8.63 \times 10^{-6}}{\omega_{j} T^{1}}\left\{\left(\frac{m}{M}\right)^{\frac{3}{2}} \int_{0}^{\infty} \Omega(i, j)\right. \\
\left.\quad \times \exp \left(-M v_{j}{ }^{2} / 2 k T\right) d\left(\frac{M v_{j}{ }^{2}}{2 k T}\right)\right\} \mathrm{cm}^{3} \mathrm{sec}^{-1},
\end{aligned}
$$

$M$ being the reduced mass, and $m$ the electron mass. This usually varies comparatively slowly with $T$. The corresponding excitation coefficient $q_{i j}$ is obtained from (5) and (12).

When many quantized angular momentum components $l$ are important, a classical or semiclassical treatment may be permissible. Low-energy atomelectron collisions take on a more typically quantum mechanical aspect because only a few values of $l$ are generally important. The reason for this may be understood from the fact that, for large $L=m v R, R$ must be very large when $m$ and $v$ are both small; such remote encounters are not likely to produce transitions.

Because of the indistinguishability of electrons, direct and exchange excitation cannot, in general, be

${ }^{1}$ M. H. Hebb and D. H. Menzel, Astrophys. J. 92, 408 (1940). regarded as distinct physical processes. However, when the total spin is well defined and is different in the initial and final atomic states, the transition can take place only by exchange.

For collisions between electrons and neutral atoms the collision strengths $\Omega$ tend to zero at the threshold. Due to the attractive field in collisions between electrons and positive ions the electron may have considerable velocity in the immediate vicinity of the ion even if its velocity tends to zero at infinity. For this reason the corresponding collision strengths tend to finite limits at thresholds and may be treated as constants for sufficiently slow collisions; the quantity in braces in (12) may then be equated to $\Omega$.

\section{Scattering Matrix}

Let $\psi_{\alpha}\left(\mathbf{r}_{A}\right)$ be an atom wave function satisfying the Schroedinger equation

$$
H_{A} \psi_{\alpha}\left(\mathbf{r}_{A}\right)=E_{\alpha} \psi_{\alpha}\left(\mathbf{r}_{A}\right)
$$

$H_{A}$ being the atom Hamiltonian. Consider that incoming electrons with angular momentum quantum numbers $l^{\prime}, m^{\prime}$ are incident on an atom in state $\alpha^{\prime}$ and that outgoing electrons are associated with $\alpha^{\prime}$ and with all other states $\alpha$ of the atom. The appropriate wave function is then

$$
\Psi\left(\gamma^{\prime} \mid \mathbf{r}_{A} \mathbf{r}\right)=\sum_{\gamma} \psi_{\gamma}\left(\mathbf{r}_{A}, \hat{r}\right)-F_{r}\left(\gamma^{\prime} \mid r\right),
$$

where $\gamma=(\alpha, l, m), \psi_{\gamma}\left(\mathbf{r}_{A}, \hat{r}\right)=\psi_{\alpha}\left(\mathbf{r}_{A}\right) Y_{l m}(\hat{r}) \quad\left(Y_{l m}\right.$ being a normalized spherical harmonic) and where, for $r \rightarrow \infty$,

$$
F_{\gamma}\left(\gamma^{\prime} \mid r\right) \sim \frac{1}{k_{\alpha^{\frac{2}{2}}}}\left[\delta\left(\gamma, \gamma^{\prime}\right) e^{-i\left(k r-\frac{1}{2} l \pi\right)}-\mathbf{S}\left(\gamma, \gamma^{\prime}\right) e^{+i\left(k r-\frac{1}{2} l \pi\right)}\right] .
$$

The probability that the atom is excited to state $\alpha$ and that the outgoing electron has angular momentum $l m$ is

$$
\Omega^{l m, l^{\prime} m^{\prime}}\left(\alpha, \alpha^{\prime}\right)=\left|\mathbf{S}\left(\alpha l m, \alpha^{\prime} l^{\prime} m^{\prime}\right)\right|^{2}
$$

and the total collision strength is

$$
\Omega\left(\alpha, \alpha^{\prime}\right)=\sum_{l m l^{\prime} m^{\prime}}\left|\mathbf{S}\left(\alpha l m, \alpha^{\prime} l^{\prime} m^{\prime}\right)\right|^{2} .
$$

When electron spin is included and properly antisummetrized wave functions are used, ${ }^{2(b)}$ (15) is replaced by

$$
\Omega\left(\alpha, \alpha^{\prime}\right)=\frac{1}{2} \sum_{l m i m_{s} l^{\prime} m l^{\prime} m_{s}^{\prime}}\left|\mathbf{S}\left(\alpha l m_{l} m_{s}, \alpha^{\prime} l^{\prime} m_{l}^{\prime} m_{s}^{\prime}\right)\right|^{2},
$$

2 (a) M. J. Seaton, Proc. Roy. Soc. (London) A208, 408 (1951); (b) Phil. Trans. A245, 469 (1953); (c) Proc. Roy. Soc. (London) A218, 400 (1953); (d) Ann. Astrophys. 17, 550 (1954); (e) The Airglow and the Aurorae, edited by E. B. Armstrong and A. Dalgarno (Pergamon Press, London, 1955), p. 289; (f) Ann. Astrophys. 18, 188 (1955); (g) ibid. 18, 206 (1955); (h) Compt. rend. 240, 1317 (1955); (i) Monthly Notices Roy. Astron. Soc. 115, 279 (1955); (j) Proc. Phys. Soc. (London) A68, 457 (1955); (k) Proc. Roy. Soc. (London) A231, 37 (1955); (l) Proc. Phys. Soc. (London) A70, 620 (1957). 
the factor $\frac{1}{2}$ being introduced on averaging over the initial spin orientation (quantum number $m_{s}$ ) of the colliding electron. The conservation theorem follows from $\mathbf{S}$ being unitary, $\sum_{\gamma}\left|\mathbf{S}\left(\gamma, \gamma^{\prime}\right)\right|^{2}=1$.

The wave function $\Psi$ should be a solution of the Schroedinger equation $(H-E) \Psi=0$. Suppose that an approximate solution $\Psi^{t}$ has been obtained with scattering matrix $\mathbf{S}$. It was shown by $\mathrm{Kohn}^{3}$ that an improved estimate for $\mathbf{S}$ is

$\mathbf{S}\left(\gamma, \gamma^{\prime}\right)=\mathbf{S}^{t}\left(\gamma, \gamma^{\prime}\right)+\frac{i m}{\hbar^{2}} \int \bar{\Psi}^{t}(\gamma)[H-E] \bar{\Psi}^{t}\left(\gamma^{\prime}\right) d \mathbf{r}_{A} d \mathbf{r}$,

$\bar{\Psi}^{t}$ being a conjugate function for the final state. The error in $\mathbf{S}$ calculated from (17) will be of quadratic order in the errors in the wave functions* $\Psi^{t}, \bar{\Psi}^{t}$.

For practical calculations the Schroedinger equation is replaced by a set of coupled equations of the type ${ }^{2(b)}$

$$
\left[\frac{d^{2}}{d r^{2}}-\frac{l(l+1)}{r^{2}}+k_{\alpha}^{2}\right] F_{\gamma}=\sum_{\gamma^{\prime}}\left[U_{\gamma \gamma^{\prime}}-X_{\gamma \gamma^{\prime}}\right] F_{\gamma^{\prime}}
$$

where $U_{\gamma \gamma^{\prime}}$ is an interaction potential and $X_{\gamma \gamma^{\prime}}$ an exchange operator:

$$
X_{\gamma \gamma^{\prime}} F_{\gamma}(r)=\int_{0}^{\infty} K_{\gamma \gamma^{\prime}}\left(r, r^{\prime}\right) F_{\gamma}\left(r^{\prime}\right) d r^{\prime}
$$

When these equations are satisfied the correction term in (17) is zero.

The distorted wave (DW) approximation is obtained on solving

$$
\left[\frac{d^{2}}{d r^{2}}-\frac{l(l+1)}{r^{2}}+k_{\alpha}{ }^{2}\right] F_{\gamma}{ }^{t}=V_{\gamma \gamma} F_{\gamma}{ }^{t}
$$

and substituting the solutions in (17). For $\gamma \neq \gamma^{\prime}$ this gives

$$
\mathbf{S}\left(\gamma, \gamma^{\prime}\right)=\frac{i}{2} \int F_{\gamma^{t}}(\gamma \mid r)\left[U_{\gamma \gamma^{\prime}}-X_{\gamma \gamma^{\prime}}\right] F_{\gamma^{\prime}}\left(\gamma^{\prime} \mid r\right) d r .
$$

The Born approximation is obtained by neglecting the right-hand side of (20) and neglecting $X_{\gamma \gamma^{\prime}}$ in (21). The assumptions made in the Born approximation are (i) the distortion is small, (ii) the coupling is weak, and (iii) exchange can be neglected.

\section{Quantum Defect Method}

The bound state energy levels of a neutral atom form infinite sequences converging to spectral limits in which the ion is left in a state of definite energy. The corresponding positive energy states represent scatter-

\footnotetext{
${ }^{3}$ W. Kohn, Phys. Rev. 74, 1763 (1948).

* It is assumed that exact atomic wave functions are used; an additional error will result from using approximate atomic functions.
}

ing of an electron by a positive ion. It might be expected that the scattering matrix for slow collisions could be deduced by extrapolating information obtained from a study of the bound states. The theory developed so $\mathrm{far}^{2(\mathrm{~h})(1), 4}$ is directly applicable only to the problem of elastic scattering but may be used in certain cases to obtain empirical estimates of inelastic scattering cross sections.

Let $I_{n l}$ be the energy required to remove electron $n l$ and $-2 Z / r$ be the asymptotic form of the potential $U(r)$ in which it moves, both measured in rydbergs $\left(13.60 \mathrm{ev}\right.$ or $\left.109737 \mathrm{~cm}^{-1}\right)$. Then $I_{n l}=Z^{2} / n_{l}{ }^{* 2}$ defines the effective quantum number $n_{l}{ }^{*}$ and $\mu_{l}\left(-I_{n l}\right)=n_{l}{ }^{*}$ $-n$ defines the quantum defect $\mu_{l}$. For the corresponding continuum the phase shift $\delta_{l}\left(k^{2}\right)$ is defined in terms of the asymptotic behavior of the wave function:

$$
\begin{aligned}
F(k, l, Z ; r) \sim k^{-\frac{1}{2}} \sin [ & k r-\frac{1}{2} l \pi+\frac{Z}{k} \ln (2 k r) \\
& \left.+\arg \Gamma\left(l+1-i \frac{Z}{k}\right)+\delta_{l}\left(k^{2}\right)\right] .
\end{aligned}
$$

For the pure Coulomb field, $U(r)=-2 Z / r$, both phase shifts $\delta_{l}$ and quantum defects $\mu_{l}$ are zero.

For $k \ll 2 \pi Z$ it may be shown that

$$
\delta_{l}\left(k^{2}\right)=\pi \mu_{l}\left(k^{2}\right),
$$

where $\mu_{l}\left(k^{2}\right)$ is the quantum defect extrapolated as a function of the energy. Use of this relation is referred to as the quantum defect method.

\section{COLLISIONS BETWEEN HEAVY PARTICLES}

Inelastic collisions between particles of atomic mass have been discussed in a recent review by Bates and Dalgarno. ${ }^{5}$ Here we consider certain processes of particular importance for studies of interstellar matter.

\section{Collisional Excitation of the hfs States of $\mathrm{H} 1 \mathrm{~s}$}

The hfs states of $\mathrm{H} 1 s$ have $F=0$ or $F=1$, where $F$ is the total spin quantum number. Let the number density of $\mathrm{H}$ atoms in these states be $n_{0}$ and $n_{1}$ and let the total hydrogen density be $n=\left(n_{0}+n_{1}\right)$. The ratio $n_{0} / n_{1}$ is determined from $21-\mathrm{cm}$ observations with the spin temperature $T_{S}$ defined by the Boltzmann relation (3). Purcell and Field ${ }^{6}$ have discussed the relation between the spin temperature, the kinetic temperature $T_{H}$, and the radiation temperature $T_{R}$ (defined by expressing the energy density of background radiation at the frequency of the $21-\mathrm{cm}$ line as a Planck function

\footnotetext{
4 F. S. Ham, Solid State Physics, edited by F. Seitz and D. Turnbull (Academic Press, Inc., New York, 1955), Vol. 1, p. 127.

5 D. R. Bates and A. Dalgarno, The Airglow and the Aurorae. edited by E. B. Armstrong and A. Dalgarno (Pergamon Press, London, 1955), p. 328.

${ }^{6}$ F. M. Purcell and G. B. Field, Astrophys. J. 124, 542 (1956).
} 


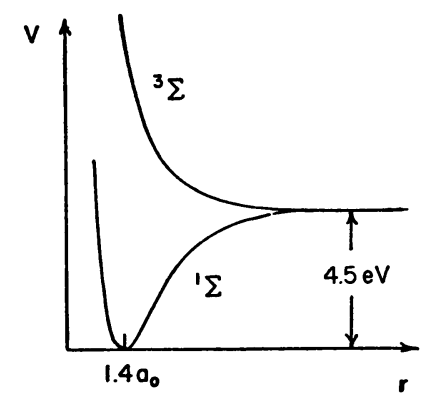

FIG. 2. Interaction potentials for collisions between two $\mathrm{H}$ atoms.

$\left.\rho_{\nu}\left(T_{R}\right)\right)$. The equation defining a steady state for the hfs level populations is

$n_{1}\left[n q_{10}+A_{10}+B_{10 \rho_{\nu}}\left(T_{R}\right)\right]=n_{0}\left[n q_{01}+B_{01} \rho_{v}\left(T_{R}\right)\right]$,

where $q_{F F^{\prime}}$ is the rate coefficient for

$$
\mathrm{H}+\mathrm{H}(F) \rightarrow \mathrm{H}+\mathrm{H}\left(F^{\prime}\right),
$$

$A_{10}=2.85 \times 10^{-15} \mathrm{sec}^{-1}$ is the spontaneous emission probability, $B_{01}$ the Einstein absorption coefficient and $B_{10}$ the Einstein stimulated emission coefficient. From (24) Purcell and Field obtain

with

$$
T_{S}=\frac{x n}{1+x n} T_{H}+\frac{1}{1+x n} T_{R},
$$

$$
x=h \nu q_{10} / k T_{H} A_{10} \text {. }
$$

A change in atom spin can be grought about during collision only by exchange of electrons. The two atoms may approach either along a repulsive ${ }^{3} \sum$ potential curve or an attractive ${ }^{1} \sum$ potential curve (Fig. 2). In the former case the distance of closest approach will be large and the exchange probability small; but along the attractive curve the atoms may approach much more closely, and the average exchange probability will be close to one-half. The potential curves of Fig. 2 may be used without modification for states of zero relative angular momentum. With impact parameter $R$, and initial relative velocity $v$, the angular momentum is $L=M v R$; and the effective potential becomes $V^{\prime}=V+L^{2} / 2 M r^{2}=V+E R^{2} / r^{2}$, where $E=M v^{2} /$ 2. For given kinetic energy $E$ the centrifugal force gives rise to a potential barrier (Fig. 3) which increases

$$
\left(V+E R^{2} / r^{2}\right)
$$

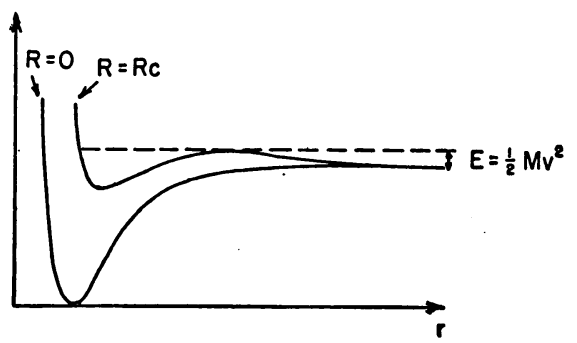

Fig. 3. $\mathrm{H}-\mathrm{H}$ collisions: attractive ${ }^{1} \Sigma$ potential plus repulsive centrifugal potential. with increasing $R$. According to classical theory, for $R$ greater than a critical value $R_{c}$ the colliding particles will be unable to penetrate the barrier; the exchange probability will then be small. For collisions along the attractive curve it may therefore be expected that the exchange cross section should be close to $\pi R_{c}{ }^{2} / 2$. More complete analysis by ${ }^{7}$ Purcell and Field shows that the spin-change cross section $Q(1,0)$ is equal to $\pi R_{c}{ }^{2} / 8$.

The results obtained by Purcell and Field are given in Table I. Putting $\left(M v R_{c}\right)^{2}=\hbar^{2} l_{c}\left(l_{c}+1\right)$, a condition for the validity of their semiclassical treatment is that $l_{c}$ should be large; values given in Table I show that the results will be less reliable at the lowest temperature considered. Purcell and Field point out that the gas-kinetic cross section for collision of two $\mathrm{H}$ atoms will be close to $8 Q(1 \rightarrow 0)$.

With $n>1$ and $T_{R}$ not too large $T_{S}$ will be nearly equal to $T_{H}$, but exceptional conditions may occur for which this is no longer the case.

\section{Cooling of the Interstellar Gas by Excitation of Molecular Rotation}

Excitation of $\mathrm{H}_{2}$ rotational levels may occur through atom-molecule collisions,

$$
\mathrm{H}+\mathrm{H}_{2}(J=0) \rightarrow \mathrm{H}+\mathrm{H}_{2}(J=2),
$$

TABLE I. Cross sections for excitation of the hfs states of $\mathrm{H} 1 s$.

\begin{tabular}{rrrr}
\hline \hline$T_{H}$ & $\bar{Q}(1,0)$ & & \\
$\left({ }^{\circ} \mathrm{K}\right)$ & $\left(\pi a 0^{2}\right)$ & $l_{c}$ & $x$ \\
\hline 10 & 13.5 & 3 & 190 \\
100 & 8.0 & 7 & 35 \\
1000 & 4.9 & 17 & 6.7 \\
\hline
\end{tabular}

or through molecule-molecule collisions,

$$
\mathrm{H}_{2}(J=0)+\mathrm{H}_{2}(J=0) \rightarrow \mathrm{H}_{2}(J=0)+\mathrm{H}_{2}(J=2) .
$$

Cross sections for the $\mathrm{H}_{2}-\mathrm{H}_{2}$ case, which will be the most important when $n\left(\mathrm{H}_{2}\right)$ greatly exceeds $n(\mathrm{H})$, may be deduced from results of ultrasonic dispersion measurements. ${ }^{8}$

Lacking further information on the $\mathrm{H}-\mathrm{H}_{2}$ case, which is likely to be the more important in typical interstellar clouds, Spitzer ${ }^{9}$ assumed the cross section to be the same as that for $\mathrm{H}_{2}-\mathrm{H}_{2}$. Improved estimates of the cooling rate may now be obtained using the results of recent calculations by Takayanagi ${ }^{10}$ for (28) and (29).

The following expression is used for the $\mathrm{H}-\mathrm{H}_{2}$ and $\mathrm{H}_{2}-\mathrm{H}_{2}$ interaction potential ${ }^{11,12}$ (see Fig. 4):

$$
V=D e^{-2 \alpha\left(r-r_{0}\right)}\left[1-2 e^{\alpha\left(r-r_{0}\right)}+\beta P_{2}(\cos \chi)\right] .
$$

${ }^{7}$ See also J. P. Wittke and R. H. Dicke, Phys. Rev. 103, 620 (1956).

8 J. E. Rhodes, Phys. Rev. 70, 932 (1946).

${ }^{9}$ L. Spitzer, Astrophys. J. 109, 337 (1949).

${ }^{10} \mathrm{~K}$. Takayanagi, Proc. Phys. Soc. (London) A70, 348 (1957).

${ }^{11}$ H. Margenau, Phys. Rev. 63, 131 (1943).

12 A. A. Evett and H. Margenau, Phys. Rev. 90, 1021 (1953). 
For $V$ in atomic units the parameters in (30) are given in Table II. It is seen that $D, r_{0}$, and $\alpha$ are very similar for the two cases but that the coefficient $\beta$, determining the anisotropy of the potential, is much larger for $\mathrm{H}_{2}-\mathrm{H}$ than for $\mathrm{H}_{2}-\mathrm{H}_{2}$. The wave functions of relative motion are calculated neglecting the term $\beta P_{2}(\cos \chi)$, which is then treated as the coupling term leading to rotational transitions (compare the DW method of Sec. II 4). Since a number of partial waves of different $l$ have to be considered, the calculations are simplified by using the modified wave-number method ${ }^{13}$ which employs an effective centrifugal potential $-\hbar^{2} l(l+1) / 2 M \tilde{r}^{2}$ independent of $r$. Takayanagi obtains for the cross sections, averaged over Maxwellian velocity distributions,

$$
\bar{Q}(0,2)=\beta^{2} G(T) \pi \bar{r}^{2},
$$

where $G(T)$ is given as a function of kinetic temperature $T_{H}$ in Table III.

The value of $\bar{r}$ should be comparable with the molecular dimensions. Adopting $\bar{r}=5$ atomic units for $\mathrm{H}_{2}-\mathrm{H}_{2}$, Takayanagi obtains $\bar{Q}(0,2)=2.8 \times 10^{-2} \quad a_{0}{ }^{2}$ for $T=197.7^{\circ} \mathrm{K}$ compared with $5.2 \times 10^{-2} a_{0}{ }^{2}$ from the ultrasonic dispersion measurements. For this type of

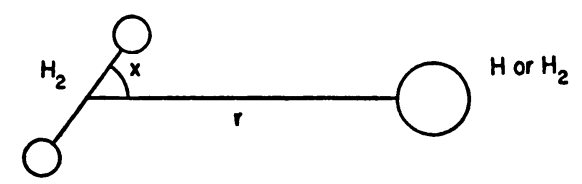

Fig. 4. For the $\mathrm{H}_{2}-\mathrm{H}_{2}$ case the potential is averaged over all orientations of the second molecule.

calculation the agreement may be regarded as satisfactory.

According to these calculations the cross section for $\mathrm{H}_{2}-\mathrm{H}$ should exceed that for $\mathrm{H}_{2}-\mathrm{H}_{2}$ by a factor of about 10, this factor being determined almost entirely by the ratio of the values of $\beta^{2}$ for the two cases (the similarity of the potentials suggests that similar values of $\bar{r}$ should be used). Takayanagi has also considered $\mathrm{H}_{2}-\mathrm{H}_{2}$ collisions in which both molecules are excited to the $J=2$ state, and $\mathrm{H}_{2}-\mathrm{H}$ collisions in which the molecule is excited to the $J=4$ state. He concludes that such processes will be less important than (28), (29) for $T<10^{3} \mathrm{~K}$. The para-ortho transitions with $\Delta J=1$ involve a change in the total nuclear spin eigenfunctions and will therefore have probabilities much less than for the para-para transitions $J=0 \rightarrow 2$.

When $n(\mathrm{H})>n\left(\mathrm{H}_{2}\right)$, and $n(\mathrm{H})$ is small, the cooling rate due to excitation of molecular rotation should therefore be ten times greater than that estimated by Spitzer. When $n(\mathrm{H})$ exceeds a certain critical value [inversely proportional to $\bar{Q}(0,2)]$ collisions produce a Boltzmann distribution among the rotational levels,

${ }^{13} \mathrm{~K}$. Takayanagi and S. Kaneko, Sci. Rept. Saitama Univ. A1, 111 (1954).
TABLE II. Parameters in the $\mathrm{H}_{2}-\mathrm{H}$ and $\mathrm{H}_{2}-\mathrm{H}_{2}$ potentials.

\begin{tabular}{|c|c|c|}
\hline & $\mathrm{H}_{2}-\mathrm{H}$ & $\mathrm{H}_{2}-\mathrm{H}_{2}$ \\
\hline $\begin{array}{l}D \\
r_{0} \\
2 \alpha \\
\beta\end{array}$ & $\begin{array}{l}1.0 \times 10^{-4} \\
6.3 \\
1.87 \\
0.26\end{array}$ & $\begin{array}{l}1.1 \times 10^{-4} \\
6.4 \\
1.87 \\
0.075\end{array}$ \\
\hline
\end{tabular}

and the cooling rate is then determined by the abundance of molecules and the radiative transition probabilities, but does not depend on $n(\mathrm{H})$ or on the cross sections. Quite generally the revised cooling rate may be written

$$
L=\frac{10 n(\mathrm{H}) n\left(\mathrm{H}_{2}\right) \Upsilon_{1} \Upsilon_{2}}{10 n(\mathrm{H}) \Upsilon_{2}+\Upsilon_{1}} \operatorname{ergs~cm}^{-3} \mathrm{sec}^{-1},
$$

where $\Upsilon_{1}$ and $\Upsilon_{2}$ are the functions tabulated by Spitzer.

\section{H $2 s \rightarrow 2 p$ Transitions Produced by Proton and by Electron Impact}

The two-quantum process, ${ }^{14,16,2(i)}$

$$
\mathrm{H}(2 s) \rightarrow \mathrm{H}(1 s)+h \nu_{1}+h \nu_{2},
$$

will give an important contribution to the continuum intensities of gaseous nebulae, provided that the probability of collisions

$$
\mathrm{H}(2 s)+A \rightarrow \mathrm{H}(2 p)+A
$$

followed by emission of Lyman- $\alpha$ is not large compared to the radiative transition probability for (33), $A=\mathbf{8 . 2 2 7}$ $\mathrm{sec}^{-1}$. Purcell ${ }^{16}$ has shown that the most important collisions will be

$$
\mathrm{H}(2 s)+\mathrm{H}^{+} \rightarrow \mathrm{H}(2 p)+\mathrm{H}^{+}
$$

although similar collisions with electrons also have to be taken into account. Purcell calculated the transition probabilities $P(R)$ by the method described in Sec. II2 and applied a correction to insure that the conservation condition (8) was satisfied for all $R$. In view of the importance of (35) indicated by Purcell's result a quantum mechanical study of the problem has been made. ${ }^{2(j)}$ It is possible to formulate an exactly soluble

\begin{tabular}{|c|c|c|c|}
\hline$T_{\boldsymbol{H}}\left({ }^{\circ} \mathbf{K}\right)$ & $\mathrm{H}_{2}-\mathrm{H}$ & $G(T)$ & $\mathrm{H}_{2}-\mathrm{H}_{2}$ \\
\hline $\begin{array}{r}100 \\
200 \\
1000\end{array}$ & $\begin{array}{l}0.003 \\
0.047 \\
1.45\end{array}$ & & $\begin{array}{l}0.003 \\
0.063 \\
1.99\end{array}$ \\
\hline
\end{tabular}
problem in which the essential features of the problem presented by the reaction (35) are retained. The solutions for $\Omega^{l}$ are shown in Fig. 5 ( $l$ is so large that

TABLE III.

${ }^{14}$ L. Spitzer and J. L. Greenstein, Astrophys. J. 114, 407 (1951).

${ }^{15}$ A. Y. Kipper, Tartu Astron. Obs. Publ. 32, 63 (1952).

${ }^{16}$ E. M. Purcell, Astrophys. J. 116, 457 (1952) 


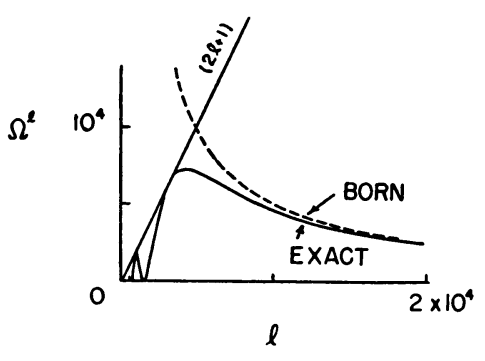

Fig. 5. Collision strengths obtained from exactly soluble equations resembling the collision equations for $\mathrm{H}(2 s)+\mathrm{H}^{+}$ $\rightarrow \mathrm{H}(2 p)+\mathrm{H}^{+}$. The maximum value of $\Omega^{l}$ consistent with the conservation theorem is $(2 l+1)$.

it may be represented as a continuous variable). The Born approximation gives good results for large $l$ but leads to a violation of the conservation theorem (10) for small $l$. Defining $l_{0}$ by

$$
\Omega^{l_{0}} \text { Born }=\frac{1}{2}\left(2 l_{0}+1\right)
$$

it is found that the Born approximation is satisfactory for $l>l_{0}$ and that little error is introduced on putting $\Omega^{l}=\frac{1}{2}(2 l+1)$ for $l<l_{0}$. For a reaction such as (35), $\boldsymbol{\Omega}^{l}{ }_{\text {Born }}$ would be difficult to evaluate but, so long as $R_{l}$ (see Sec. II3) is much greater than the atomic dimensions (which is the case for $l>l_{0}$ ) the Bethe approximation, $\Omega^{l}{ }_{\text {Bethe }}$, does not differ significantly from $\Omega^{l}{ }_{B o r n}$ and may be evaluated much more easily. Defining $l_{c}$ by

$$
\begin{array}{ll}
\Omega_{\text {Bethe }}^{l} \leqslant \frac{1}{2}(2 l+1) & \text { for } l \geqslant l_{c} \\
\Omega_{\text {Bethe }}>\frac{1}{2}(2 l+1) & \text { for } l<l_{c},
\end{array}
$$

the approximation used for $\Omega(2 s, 2 p)$ is

$$
\Omega=\sum_{l=0}^{l_{c}} \frac{1}{2}(2 l+1)+\sum_{l=l_{c}+1}^{\infty} \Omega^{l_{\text {Bethe. }}}
$$

The rate coefficients obtained for proton and electron impact are given in Table IV. For equal electron and proton densities the rate of $2 s \rightarrow 2 p$ transitions per $\mathrm{cm}^{3} /$ sec is $n(e) n(H, 2 s) q(2 s \rightarrow 2 p)$. Consideration of the exactly soluble problem and of results obtained on using (38) for excitation of the $\mathrm{Na} \mathrm{D}$ lines by electron impact (Sec. IV3), which is a less favorable case than H $2 s \rightarrow 2 p$, suggests that the errors in the Table IV results should not exceed $\pm 20 \%$.

\section{Charge Transfer Reactions}

The cross section for

$$
A+B^{+} \rightarrow A^{+}+B
$$

will be large when the ionization potentials $I(A)$ and $I(B)$ are nearly equal; this is illustrated by the results of Dalgarno and Yadav $^{17}$ for

$$
\mathrm{H}^{+}+\mathrm{H} \rightarrow \mathrm{H}+\mathrm{H}^{+} \text {. }
$$

Chamberlain ${ }^{18}$ has discussed the possible importance of

$$
\mathrm{H}^{+}+\mathrm{O} \rightarrow \mathrm{H}+\mathrm{O}^{+}
$$

in the Cassiopeia radio source. Since $I(\mathrm{H})=13.595 \mathrm{ev}$

\footnotetext{
${ }^{17}$ A. Dalgarno and H. N. Yadav, Proc. Phys. Soc. (London) A66, 173 (1953).

${ }^{18} \mathrm{~J}$. W. Chamberlain, Astrophys. J. 124, 390 (1956).
}

and $I(0)=13.614 \mathrm{ev}$ it may be expected that the cross section for (41) will be of the same order as that for (40); in the temperature range $1 \times 10^{4}$ to $2 \times 10^{4}{ }^{\circ} \mathrm{K}$ Chamberlain adopts $q \sim 10^{-8} \mathrm{~cm}^{3} \mathrm{sec}^{-1}$ for (41). According to Chamberlain such an order of magnitude estimate may be sufficient to establish that (41) is the dominant process; this being so, the ionization ratio $n(\mathrm{O}) / n\left(\mathrm{O}^{+}\right)$is determined by considerations of detailed balancing.

\section{Excitation of Atoms by Impact of Thermal Protons}

Cross sections for excitation of neutral atoms by proton impact will be of similar magnitude to those for electron impact when the velocities of relative motion are the same (see Sec. II2). The electron excitation cross sections rise steeply just beyond the threshold and have maxima at energies about twice the threshold energy. For proton impact the energies at which the cross-section maxima occur will therefore be much

\begin{tabular}{|c|c|c|}
\hline & $T=10^{4}{ }^{\circ} \mathrm{K}$ & $T=2 \times 10^{4}{ }^{\circ} \mathrm{K}$ \\
\hline Protons $\left\{\begin{array}{l}10^{4} \times q\left(2 s \rightarrow 2 p_{3}\right) \\
10^{4} \times q\left(2 s \rightarrow 2 p_{\frac{3}{2}}\right)\end{array}\right.$ & $\begin{array}{l}2.51 \\
2.23\end{array}$ & $\begin{array}{l}2.08 \\
2.19\end{array}$ \\
\hline Electrons $\left\{\begin{array}{l}10^{4} \times q\left(2 s \rightarrow 2 p_{1}\right) \\
10^{4} \times q\left(2 s \rightarrow 2 p_{3}^{3}\right)\end{array}\right.$ & $\begin{array}{l}0.22 \\
0.35\end{array}$ & $\begin{array}{l}0.17 \\
0.27\end{array}$ \\
\hline $10^{4} \times q(2 s \rightarrow 2 p)$ & 5.3 & 4.7 \\
\hline
\end{tabular}
greater and the cross sections at thermal energies will be small. $\dagger$ Thus, for the reaction

$$
\mathrm{O}\left({ }^{1} D\right)+\mathrm{H}^{+} \rightarrow \mathrm{O}\left({ }^{1} S\right)+\mathrm{H}^{+},
$$

TABLE IV. $\mathrm{H} 2 s-2 p$ transitions produced by electron and by proton impact.

the rate coefficient at thermal energies is at most a few percent of that for excitation by electron impact. ${ }^{2(d)}$

Due to the repulsive Coulomb field, cross sections will be minute for transitions in positive ions produced by impact of thermal protons.

\section{Chemical Processes}

Little can be added to the discussions by Bates and and Spitzer ${ }^{19}$ and by Herzberg ${ }^{20}$ of the reactions leading to formation and destruction of interstellar molecules. The most uncertain single quantity is the $f$ value for $\mathrm{CH} \lambda 4300$.

\section{INELASTIC COLLISIONS BETWEEN ATOMS AND ELECTRONS}

Experimental and theoretical work on atom-electron collisions has been discussed in two recent review articles by Massey. ${ }^{21}$

$\dagger$ In the case of $\mathrm{H} 2 s \rightarrow 2 p$ the large excitation rate for proton impact may be understood as a consequence of the threshold energies being very small.

${ }_{19}$ D. R. Bates and L. Spitzer Astrophys. J. 113, 441 (1951).

${ }^{20}$ G. Herzberg, Mém. soc. roy. sci. Liège 15, 291 (1955).

${ }^{21}$ H. S. W. Massey, Handbuch der Physik (Springer-Verlag, Berlin, 1956), Vol. 36, p. 307; Revs. Modern Phys. 28, 199 (1956). 


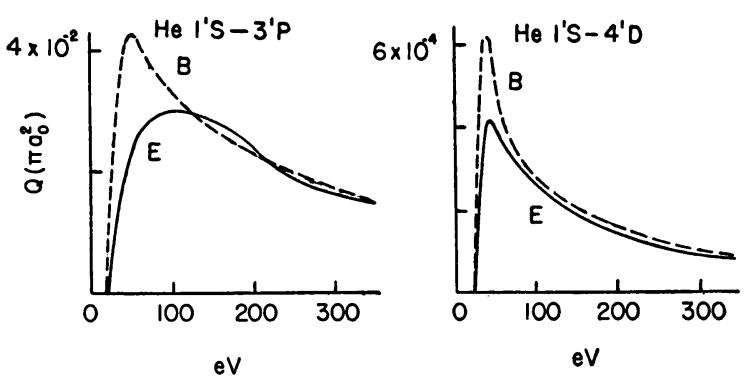

Fig. 6. The Born approximation $(B)$ for excitation of $\mathrm{He}$ by electron impact. Experimental cross sections $(E)$ measured in arbitrary units have been fitted to the Born curves at high energies.

\section{Born Approximation}

Although correctly considered a high-energy approximation, the Born method may often be used to obtain low-energy results which will be good to within better than an order of magnitude. It is usually better for optically forbidden transitions not involving a change of spin than for optically allowed transitions. Figure 6 shows some typical results for transitions in $\mathrm{He}$ and Fig. 7 some ionization cross sections. ${ }^{22} \mathrm{~A}$ modified form of the Born approximation has been used by Geltman $^{23}$ to obtain $\mathrm{H}$ and $\mathrm{He}$ ionization cross sections near threshold; his $\mathrm{He}$ results are about one-half of those obtained experimentally.

Rate coefficients for $\mathrm{H}$ excitation have been calculated by Chamberlain ${ }^{24}$ using Born cross sections for $1 s \rightarrow 2 s$, $2 p, 3 s, 3 p$, and $3 d$ and estimates for $n>3$. Born cross sections for excitation of higher excited states of $\mathrm{H}$ and for ionization are given by McCarroll. ${ }^{25}$ Born cross sections have been calculated by Moiseiwitsch ${ }^{26}$ for transitions from $\mathrm{He} 2^{3} S$.

Born's approximation cannot be used for spin change transitions. Such exchange processes depend on the overlap between atomic wave functions and the wave functions of the colliding electron, and therefore involve much more refined properties of the collision wave functions than those required when the Born approximation is successful. In consequence Oppenheimer's exchange modification of the Born approximation is of little practical use. The Born approximation also fails completely for slow collisions between electrons and positive ions since it gives cross sections with incorrect energy dependence.

\section{Distorted Wave Approximation}

In some cases the main defect in the Born approximation may be neglect of distortion of the wave functions of relative motion by the atomic field. In such cases

\footnotetext{
22 These figures are taken from Bates, Fundaminsky, Leech, and Massey, Phil. Trans. Roy. Soc. A243, 93 (1950).

${ }^{23}$ S. Geltman, Phys. Rev. 102, 171 (1956).

24 J. W. Chamberlain, Astrophys. J. 117, 387 (1952).

${ }_{25}$ R. McCarroll, Proc. Phys. Soc. (London) A70, 460 (1957).

${ }^{26}$ B. L. Moiseiwitsch, Monthly Notices Roy. Astron. Soc. 117, 189 (1957).
}
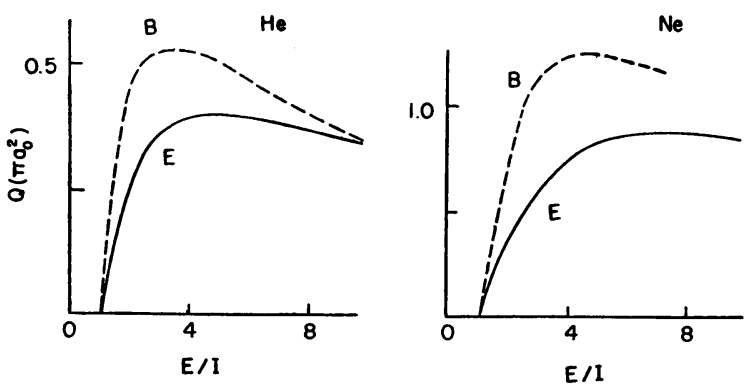

Fig. 7. Comparison of Born cross sections $(B)$ with absolute experimental measurements $(E)$ for ionization. $(E / I)$ is the ratio of the incident electron energy to the threshold ionization energy.

improved results are obtained with the DW approximation. This method, including important results obtained for $\mathrm{H}$ and $\mathrm{He}$ excitation, is discussed fully in the review articles by Massey. ${ }^{21}$

\section{Strong Coupling in Optically Allowed Transitions}

For optically allowed transitions for which the line strength ${ }^{27}$ is large, the main defect in the Born approximation may be the assumption of weak coupling.

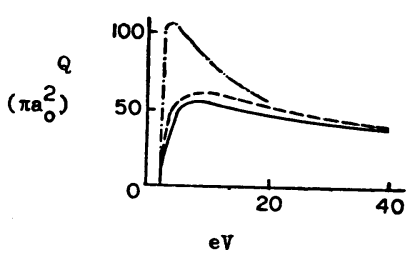

Fig. 8. Cross sections for $\mathrm{Na} 3 s \rightarrow 3 p$ produced by electron impact. Experimental, - ; Born approximation, - - - - - ; strong coupling approximation [Eq. (38) ], - - .

In such cases the strong coupling formula (38) may be used. Figure 8 shows results obtained ${ }^{2(j)}$ for $\mathrm{Na} 3 s \rightarrow 3 p$. The same method has been used by Moiseiwitsch ${ }^{26}$ for He $2^{3} S \rightarrow 2^{3} P$. It would be of interest to apply a similar method, but with allowance for Coulomb distortion, to excitation of the $\mathrm{Ca}^{+} \mathrm{H}$ and $\mathrm{K}$ lines.

\section{Excitation of Forbidden Lines in $p^{q}$ Configurations}

The configurations $2 p^{q}$ and $3 p^{q}$, with $q=2,3$, and 4 , contain the following spectral terms, conveniently denoted by $n=1,2$, and 3 :

$\begin{array}{ccc}n & q=2 \text { and } 4 & q=3 \\ 1 & { }^{3} P & { }^{4} S \\ 2 & { }^{1} D & { }^{2} D \\ 3 & { }^{1} S & { }^{2} P .\end{array}$

${ }^{27}$ E. U. Condon and G. H. Shortley, The Theory of Atomic Spectra (Cambridge University Press, New York, 1951), p. 98. 
TABLE V. Deactivation coefficients for $\mathrm{O}^{0}$ and $\mathrm{N}^{0}$ $\left(q_{n n^{\prime}}\right.$ in $\left.\mathrm{cm}^{3} \mathrm{sec}^{-1}\right)$.

\begin{tabular}{ccccccc}
\hline \hline & & $\mathrm{O}^{0}$ & & & $\mathrm{~N}^{0}$ \\
$T_{e}$ & ${ }^{0} 0^{0} q_{21}$ & $1^{0} q_{81}$ & $1^{9} q_{32}$ & $1^{9} q_{21}$ & $10^{0} q_{31}$ & $1^{0} q_{82}$ \\
\hline $5 \times 10^{2}$ & 0.9 & 0.8 & 0.4 & 0.5 & 0.4 & 0.9 \\
$1 \times 10^{3}$ & 1.6 & 1.2 & 0.6 & 0.8 & 0.6 & 1.5 \\
$5 \times 10^{3}$ & 5.0 & 3.5 & 1.9 & 2.6 & 1.8 & 4.4 \\
$1 \times 10^{4}$ & 6.7 & 5.1 & 3.0 & 3.5 & 2.7 & 6.9 \\
$5 \times 10^{4}$ & 7.6 & 7.2 & 5.6 & 4.0 & 3.7 & 13 \\
$1 \times 10^{5}$ & 6.8 & 6.6 & 6.0 & 3.5 & 3.4 & 14 \\
\hline \hline
\end{tabular}

Excitation of these terms by electron impact produces most of the forbidden line emission in gaseous nebulae.

For neutral atomic oxygen $\left(\mathrm{O}^{0}\right)$ the coupling is very strong, and the DW approximation gives results ${ }^{28}$ exceeding the conservation theorem maximum by a large factor. ${ }^{22}$ The dominant coupling terms are retained in the exact resonance (ER) approximation ${ }^{2(b)}$ in which the equations (18) are simplified on neglecting all but the spherically symmetric parts of the expansions of the interelectronic potentials $1 /\left|\mathbf{r}_{i}-\mathbf{r}_{j}\right|$; the equations (18) may then be replaced by uncoupled equations. The ER approximation involves neglect of energy differences between the spectral terms. For $\mathrm{O}^{0}$ a correction for energy differences was applied and the results obtained checked by solution of the full coupled equations for one value of $k^{2}$. Calculated deactivation coefficients for $\mathrm{O}^{0}$ and estimates for $\mathrm{N}^{0}$ are given in Table V.

Born-Oppenheimer calculations $\mathrm{s}^{29}$ for $\mathrm{O}^{+2}$, and calculations allowing for Coulomb distortion ${ }^{1}$ for $\mathrm{O}^{+2}$ and for $\mathrm{O}^{+}$and $\mathrm{N}^{+}\left(\right.$Aller $^{30}$ and Aller and White ${ }^{31}$ ) give collision strengths which are too large. The DW and ER approximations have been used ${ }^{2(c)(k)}$ for calculations on $\mathrm{N}^{+}, \mathrm{O}^{+}, \mathrm{O}^{+2}, \mathrm{Ne}^{+2}$, and $\mathrm{S}^{+}$. For $\mathrm{O}^{+2}$ neglect of energy differences in the ER approximation was shown to introduce little error. The ER wave functions should be a good deal more accurate than the DW wave functions, but the $\mathbf{S}$ matrix calculated in the ER approximation must be corrected for the neglected contributions to the potentials and exchange operators $U_{\gamma \gamma^{\prime}}$ and $X_{\gamma \gamma^{\prime}}$ (Sec. II4). These corrections were originally made using expressions similar to (17) in which the ER functions were used for $\Psi^{t}$ and the DW

TABLE VI. Collision strengths for $\mathrm{O}^{+}$.

\begin{tabular}{|c|c|c|c|}
\hline$i \quad j$ & $\mathbf{\Omega}(i, j)$ & $i$ & $\Omega(i, j)$ \\
\hline 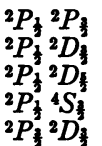 & $\begin{array}{l}0.33 \\
0.33 \\
0.38 \\
0.19 \\
0.52\end{array}$ & 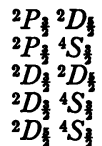 & $\begin{array}{l}0.89 \\
0.39 \\
0.85 \\
0.51 \\
0.77\end{array}$ \\
\hline
\end{tabular}

${ }^{28}$ Yamanouchi, Inui, and Amemiya, Proc. Phys. Math. Soc. Japan 22, 847 (1940).

${ }_{29}$ S. Miyamoto, Mém. Coll. Sci., Kyoto Imp. Univ. A23, 467 (1941).

${ }^{30}$ L. H. Aller, Astrophys. J. 111, 609 (1950).

${ }^{31}$ L. H. Aller and M. L. White, Astrophys. J. 54, 181 (1949), functions for $\bar{\Psi}^{t}$. Improved variational results ${ }^{32}$ for $\mathrm{O}^{+}$ have been obtained using the more accurate ER functions for both $\Psi^{t}$ and $\bar{\Psi}^{t}$ (Table VI). Previously unpublished results obtained for $\mathrm{O}^{+2}$ and $\mathrm{N}^{+}$using the same method are given in Table VII.

A partial check on the calculations may be made ${ }^{2(e), 32}$ using similar variational expressions to calculate quantum defects for the limits of various $p^{q} n l$ series. For $\mathrm{O}^{+2}$ and $\mathrm{N}^{+}$semiempirical estimates of collision strengths have been obtained (Table VII) by adjusting the parameters in the variational expressions so as to obtain good agreement with observed quantum defects. These results may be the most accurate, but this is not certain. The recommended values of Table VII are obtained on taking the mean of the variational and semiempirical results.

All available results, including estimates for cases for which calculations have not been made, are given in Table VIII. Further improvements could be made; firstly, by obtaining solutions of the full coupled equations using an electronic calculator, and secondly, by making further use of the quantum defect method.

\section{Transitions between Fine Structure Levels}

Assuming $L S$ coupling the $\mathbf{S}$ matrix is most conveniently calculated in a representation of total spin

TABLE VII. Collision strengths for $\mathrm{O}^{+2}$ and $\mathrm{N}^{+}$.

\begin{tabular}{|c|c|c|c|c|}
\hline & Previous & Variational & $\begin{array}{c}\text { Semi- } \\
\text { empirical }\end{array}$ & $\begin{array}{l}\text { Recom- } \\
\text { mended }\end{array}$ \\
\hline $\mathrm{O}^{+2}\left\{\begin{array}{l}\Omega(1,2) \\
\Omega(1,3) \\
\Omega(2,3)\end{array}\right.$ & $\begin{array}{l}1.73 \\
0.195 \\
0.61\end{array}$ & $\begin{array}{l}1.84 \\
0.255 \\
0.62\end{array}$ & $\begin{array}{l}1.34 \\
0.188 \\
0.66\end{array}$ & $\begin{array}{l}1.59 \\
0.22 \\
0.64\end{array}$ \\
\hline $\mathrm{N}^{+}\left\{\begin{array}{l}\Omega(1,2) \\
\Omega(1,3) \\
\Omega(2,3)\end{array}\right.$ & $\begin{array}{l}2.39 \\
0.223 \\
0.46\end{array}$ & $\begin{array}{l}2.63 \\
0.403 \\
0.50\end{array}$ & $\begin{array}{l}1.71 \\
0.213 \\
0.50\end{array}$ & $\begin{array}{l}2.17 \\
0.31 \\
0.50\end{array}$ \\
\hline
\end{tabular}

and total orbital angular momenta, $S^{T} L^{T}$. Then for transitions between spectral terms,

$$
\begin{aligned}
\Omega\left(\alpha S L, \alpha^{\prime} S^{\prime} L^{\prime}\right)= & \frac{1}{2} \sum_{l l^{\prime} S^{T} L^{T}}\left(2 S^{T}+1\right)\left(2 L^{T}+1\right) \\
& \times\left|\mathbf{S}\left(\alpha S L l S^{T} L^{T}, \alpha^{\prime} S^{\prime} L^{\prime} l^{\prime} S^{T} L^{T}\right)\right|^{2}
\end{aligned}
$$

To obtain $\Omega\left(\alpha S L J, \alpha^{\prime} S^{\prime} L^{\prime} J^{\prime}\right)$, we use the transformation

$$
\begin{gathered}
\mathbf{S}\left(\alpha S L J l j J^{T}, \alpha^{\prime} S^{\prime} L^{\prime} J^{\prime} l^{\prime} j^{\prime} J^{T}\right) \\
=\sum_{S^{T} L^{T}} A\left(\begin{array}{lll}
L & S & J \\
l & \frac{1}{2} & j \\
L^{T} & S^{T} & J^{T}
\end{array}\right) \mathbf{S}\left(\alpha S L l S^{T} L^{T}, \alpha^{\prime} S^{\prime} L^{\prime} l^{\prime} S^{T} L^{T}\right) \\
\times A\left(\begin{array}{lll}
L^{\prime} & S^{\prime} & J^{\prime} \\
l^{\prime} & \frac{1}{2} & j^{\prime} \\
L^{T} & S^{T} & J^{T}
\end{array}\right), \quad(44)
\end{gathered}
$$

${ }^{32}$ M. J. Seaton and D. E. Osterbrock, Astrophys. J. 125, 66 (1957). 
where $A$ is the transformation coefficient ${ }^{33}$ between $L S$ and $j j$ coupling. Then

$$
\begin{aligned}
& \Omega\left(\alpha S L J, \alpha^{\prime} S^{\prime} L^{\prime} J^{\prime}\right)=\frac{1}{2} \sum_{l l^{\prime} j j^{\prime} J T}\left(2 J^{T}+1\right) \\
& \quad \times\left|\mathbf{S}\left(\alpha S L J l j J^{T}, \alpha^{\prime} S^{\prime} L^{\prime} J^{\prime} l^{\prime} j^{\prime} J^{T}\right)\right|^{2} .
\end{aligned}
$$

These formulas have been used in calculating the $\mathrm{O}^{+}$ collision strengths given in Table VI. It should be noted that the important ratios

$$
\frac{\Omega\left({ }^{4} S,{ }^{2} D_{\frac{1}{3}}\right)}{\Omega\left({ }^{4} S,{ }^{2} D_{\frac{1}{3}}\right)}=\frac{3}{2} \quad \text { and } \quad \frac{\Omega\left({ }^{4} S,{ }^{2} P_{\frac{1}{3}}\right)}{\Omega\left({ }^{4} S,{ }^{2} P_{\frac{1}{3}}\right)}=2
$$

are determined entirely by properties of the transformation coefficients.

The collision strengths $\mathrm{C}^{+} \Omega\left(2 p_{3}, 2 p_{3}\right)$ and $\mathrm{Si}^{+}$ $\Omega\left(3 p_{1}, 3 p_{\frac{1}{3}}\right)$ are needed in order to calculate the rate of

\begin{tabular}{|c|c|c|c|c|}
\hline $\begin{array}{c}\text { Configura- } \\
\text { tion }\end{array}$ & Ion & $\Omega(1,2)$ & $\mathbf{\Omega}(1,3)$ & $\Omega(2,3)$ \\
\hline $2 p^{2}$ & $\left\{\begin{array}{l}\mathrm{N}^{+} \\
\mathrm{O}^{+2} \\
\mathrm{~F}^{+3} \\
\mathrm{Ne}^{+4}\end{array}\right.$ & $\begin{array}{l}2.17 \\
1.59 \\
1.21 \\
0.84\end{array}$ & $\begin{array}{l}0.31 \\
0.22 \\
0.17 \\
0.16\end{array}$ & $\begin{array}{l}0.50 \\
0.64 \\
0.58 \\
0.53\end{array}$ \\
\hline $2 p^{3}$ & $\left\{\begin{array}{l}\mathrm{O}^{+} \\
\mathrm{Fe}^{+2} \\
\mathrm{Ne}^{+3} \\
\mathrm{Na}^{+4}\end{array}\right.$ & $\begin{array}{l}1.28 \\
1.00 \\
0.68 \\
0.43\end{array}$ & $\begin{array}{l}0.58 \\
0.22: \\
0.23: \\
0.25:\end{array}$ & $\begin{array}{l}2.12 \\
3.11 \\
3.51 \\
3.49\end{array}$ \\
\hline $2 p^{4}$ & $\left\{\begin{array}{l}\mathrm{F}^{+} \\
\mathrm{Ne}^{+2} \\
\mathrm{Na}^{+3} \\
\mathrm{Mg}^{+4}\end{array}\right.$ & $\begin{array}{l}0.95 \\
0.76 \\
0.61 \\
0.54\end{array}$ & $\begin{array}{l}0.057 \\
0.077 \\
0.092 \\
0.112\end{array}$ & $\begin{array}{l}0.17 \\
0.27 \\
0.30 \\
0.30\end{array}$ \\
\hline $3 p^{3}$ & $\mathrm{~S}^{+}$ & 2.02 & 0.383 & 12.7 \\
\hline
\end{tabular}
cooling of the interstellar gas due to electron-ion

TABLE VIII. Collision strengths for $p^{e}$ ions. ${ }^{\mathrm{a}}$

- Estimated values are given in italic type. Values marked (: ) may be too small by a factor of about two.

collisions. Calculations may be made by purely empirical methods. In $L S$ coupling,

$$
\mathbf{S}\left(p l S^{T} L^{T}, p l S^{T} L^{T}\right)=\exp \left[2 i \pi \mu\left(p l S^{T} L^{T}, k^{2}\right)\right],
$$

where $\mu\left(p l S^{T} L^{T}, k^{2}\right)$ is the extrapolated quantum defect for the $p n l S^{T} L^{T}$ series. Neglecting a small contribution from $l \neq l^{\prime}$ the collision strengths may be calculated from (44) and (45). For the lower $p n l S^{T} L^{T}$ states of the neutral atoms, $\mathrm{C}^{0}$ and $\mathrm{Si}^{0}, L S$ coupling is a sufficiently good approximation to enable one to eliminate the spin-orbit energies by using the centres of gravity of the spectral terms. Only the differences of the quantum defects, $\mu\left(p l S^{T} L^{T}\right)-\left(p l S^{\prime T} L^{\prime T}\right)$, enter the final expression for $\Omega$. In practice, observed rather than extrapolated quantum defects have been used, this approximation being checked by using the $\mu\left(p n l S^{T} L^{T}\right)$

\begin{tabular}{|c|c|c|c|}
\hline$l$ & $\mathrm{C}^{+}$ & $\Omega^{l l}\left(p_{\left.z / 2, p_{1 / 2}\right)}\right.$ & $\mathrm{Si}^{+}$ \\
\hline $\begin{array}{c}0 \\
1 \\
2 \\
\text { estimated, } l \geq 3\end{array}$ & $\begin{array}{l}0.03 \\
0.76 \\
0.48 \\
0.01\end{array}$ & & $\begin{array}{l}0.1 \\
0.8 \\
6.5 \\
0.3\end{array}$ \\
\hline$\Omega\left(p_{\frac{3}{3}}, p_{\frac{1}{2}}\right)$ & 1.28 & & 7.7 \\
\hline
\end{tabular}

${ }^{23}$ J. M. Kennedy and M. J. Cliff, Report CRT-609, Atomic Energy of Canada Ltd., Chalk River Project (1955).
TABLE IX. Collisions strengths for $\mathrm{C}^{+}$and $\mathrm{Si}^{+}$.

and verifying that the final results for $\Omega$ are not far different for various values of $n$. The results obtained are given in Table IX. A previous calculation $\ddagger$ by the DW method for $\mathrm{C}^{+}$gave $\Omega=1.16$; for $\mathrm{Si}^{+}$a value $\Omega=6$ was estimated.

With the interstellar abundances previously assumed $^{2(f)}$ the revised cooling rate due to electron-ion collisions is

$$
\begin{array}{r}
L=10^{-23} T_{e}^{-\frac{1}{2}}\left\{0.64 e^{-92 / T_{e}}+6.4 e^{-413 / T_{e}}+1.7 e^{-554 / T_{e}}\right. \\
\left.+2.2 e^{-961 / T_{e}}\right\} n(e) n(\mathrm{H}) \mathrm{ergs} \mathrm{cm} \mathrm{cm}^{-3} \mathrm{sec}^{-1},
\end{array}
$$

$T_{e}$ being the electron temperature. Consistent with the assumed abundances, $n(e)=2 \times 10^{-4} n(\mathrm{H})$. The last two terms in the curly bracket in (48) are estimated contributions from collisions with $\mathrm{Fe}^{+}$.

\section{Elastic Collisions and Spin-Change Collisions between Electrons and Hydrogen Atoms}

When the electron temperature $T_{e}$ in the interstellar gas clouds is not equal to the kinetic temperature $T_{H}$ of the hydrogen atoms the rate of energy transfer between electrons and atoms is ${ }^{34}$

$$
\gamma=8 Q_{\mathrm{el}} \frac{m}{M_{H}}\left(\frac{2 k T_{e}}{\pi m}\right)^{\frac{1}{2}}\left(k T_{H}-k T_{e}\right),
$$

where $Q_{\mathrm{el}}$ is the cross section for elastic collisions. This cross section is also of interest in connection with the electrical conductivity of the neutral $\mathrm{H}$ gas. ${ }^{35}$ The usual quantal expression is

$$
Q_{\mathrm{el}}=\left(\pi / k^{2}\right) \sum_{l}(2 l+1)\left[\sin ^{2} \eta_{l}{ }^{+}+3 \sin ^{2} \eta_{l}^{-}\right]
$$

$\eta_{l}{ }^{+}, \eta_{l}^{-}$being the partial wave phases for the singlet and triplet spin interactions. The cross section for transitions between hyperfine structure states,

is given by

$$
\mathrm{H}(F=1)+e \rightarrow \mathrm{H}(F=0)+e,
$$

$$
Q(1,0)=\left(\pi / 4 k^{2}\right) \sum_{l}(2 l+1) \sin ^{2}\left(\eta_{l}{ }^{+}-\eta_{l}-\right) .
$$

The possible importance of (49) in the galactic halo was mentioned by Field during the Symposium discussions.

I See reference 2 (f). It may be noted that the agreement between the empirical and DW results for the individual elements of the $\mathbf{S}$ matrix is a good deal less satisfactory than the agreement between the final collision strengths.

${ }^{34}$ L. Spitzer and M. P. Savedoff, Astrophys. J. 111, 593 (1950).

${ }^{35}$ D. E. Osterbrock, Phys. Rev. 87, 468 (1952). 
TABlE X. Scattering of electrons by $\mathrm{H}$ atoms: elastic cross sections $Q_{\mathrm{el}}$ and spin-change cross sections $Q(1,0)$.

\begin{tabular}{cccccc}
\hline \hline$k$ & $Q_{\text {e1 }}$ & $Q(1,0)$ & $k$ & $Q_{\text {e1 }}$ & $Q(1,0)$ \\
\hline 0.00 & 65 & 5.5 & 0.2 & 37 & 2.5 \\
0.05 & 62 & 4.9 & 0.3 & 25 & 1.4 \\
0.10 & 54 & 4.2 & 0.4 & 18 & 0.9 \\
0.15 & 44 & 3.3 & 0.5 & 14 & 0.7 \\
\hline
\end{tabular}

- Cross sections in units of $\pi a_{0}^{2}, k^{2}$ in units of $13.60 \mathrm{ev}$.

Results of recent calculations ${ }^{36}$ are given in Table $\mathrm{X}$. The phases $\eta_{0}^{ \pm}$, calculated allowing for exchange and for polarization of the atom, are in good agreement with previous calculations $\mathbf{s}^{37-39}$; the low-energy limits for the cross sections, determined by $\left(\eta_{0}^{ \pm} / k\right)$, are unlikely to be in error by more than $\pm 15 \%$. The phases $\eta_{1}{ }^{ \pm}$, calculated allowing for exchange but neglecting polarization, are found to give small contributions to the cross sections in the range $0 \leq k \leq 0.5\left(k^{2}\right.$ in units of $13.60 \mathrm{ev})$. Recent attempts to measure the elastic scattering cross section $^{40,41}$ give results much larger than those calculated. It is not yet certain whether the discrepancy is due to the experimental results being in error or due to the effect of polarization being unexpectedly large for partial waves other than the $s$ wave.

\section{PHOTOIONIZATION CROSS SECTIONS}

Photoionization cross sections and radiative recombination coefficients are required in order to interpret observed recombination spectra and in order to predict the ionization equilibrium to be expected with a given radiation field, electron density, and electron temperature.

\section{General Formulas}

The photoionization cross section for

$$
A+h \nu \rightarrow A^{+}+e
$$

( $A$ may be an ion) is

$$
\alpha_{\nu}=\frac{8 \pi^{3} e^{2} \nu}{3 c \omega_{i}} \sum\left|\int \chi_{i}^{*}\left[\sum_{j} \mathbf{r}_{j}\right] \chi_{f}(E) d \tau\right|^{2},
$$

where $\mathbf{r}_{j}$ is the position vector of the $j$ th electron and $\chi_{i}$ is the initial bound wave function normalized to unity.

${ }^{36}$ Bransden, Dalgarno, John, and Seaton, Proc. Phys. Soc. (London) 71, 877 (1958).

${ }^{37}$ H. S. W. Massey and B. I. Moiseiwitsch, Proc. Roy. Soc. (London) A205, 483 (1951)

${ }_{38}$ T. B. Staver, Arch. Math. Naturvidenskab B51, 29 (1951)

${ }^{39}$ S. Borowitz and H. Greenberg, Bull. Am. Phys. Soc. Ser. II, 2, 172 (1957)

${ }^{40}$ Maecker, Peters, and Schenk, Z. Physik 140, 119 (1955)

11 Pederson, Malamud, and Hammer, Bull. Am. Phys. Soc. Ser. II, 2, 172 (1957).
The wave function $\chi_{f}(E)$ for $A^{+}$, together with the ejected electron, is normalized per unit energy :

$$
\int \chi_{f}^{*}\left(E^{\prime}\right) \chi_{f}(E) d \tau=\delta\left(E-E^{\prime}\right)
$$

The summation in (51) is over all initial and final states of given energy, and $\omega_{i}$ is the statistical weight of the initial level of $A$.

The recombination coefficient for the inverse of (50) is

$$
\begin{aligned}
\alpha=\frac{1}{c^{2}}\left(\frac{2}{\pi}\right)^{\frac{1}{2}}\left(m k T_{e}\right)^{-\frac{\omega_{i}}{2}} \frac{\omega_{i}}{\omega^{+}} e^{I_{i} / k T_{e}} \int_{I_{i}}^{\infty}(h \nu)^{2} a_{\nu} \\
\\
\times e^{-h \nu / k T_{e}} d(h \nu),
\end{aligned}
$$

$\omega^{+}$being the statistical weight for $A^{+}$and $I_{i}$ the threshold energy for (50).

In nebulae ionization need usually be considered only from ground states and from low-lying metastable

TABLE XI.

\begin{tabular}{cccccccc}
\hline \hline$q$ & $S L$ & $S^{\prime} L^{\prime}$ & $C$ & $q$ & $S L$ & $S^{\prime} L^{\prime}$ & $C$ \\
\hline 1 & $2 P$ & ${ }^{1} S$ & $1 / 3$ & 4 & ${ }^{3} P$ & ${ }^{4} S$ & $4 / 9$ \\
2 & $3 P$ & $2 P$ & $2 / 3$ & 4 & $3 P$ & ${ }^{2} D$ & $5 / 9$ \\
2 & $1 D$ & $2 P$ & $2 / 3$ & 4 & $3 P$ & $2 P$ & $1 / 3$ \\
2 & $1 S$ & $2 P$ & $2 / 3$ & 4 & $1 D$ & $4 S$ & 0 \\
3 & $4 S$ & $3 P$ & 1 & 4 & $1 D$ & $2 D$ & 1 \\
3 & $4 S$ & $1 D$ & 0 & 4 & $1 D$ & $2 P$ & $1 / 3$ \\
3 & $4 S$ & $1 S$ & 0 & 4 & $1 S$ & $4 S$ & 0 \\
3 & $2 D$ & $3 P$ & $1 / 2$ & 4 & $1 S$ & $2 D$ & 0 \\
3 & $2 D$ & $1 D$ & $1 / 2$ & 4 & $1 S$ & $2 P$ & $4 / 3$ \\
3 & $2 D$ & $1 S$ & 0 & 5 & $2 P$ & $3 P$ & 1 \\
3 & $2 P$ & $3 P$ & $1 / 2$ & 5 & $2 P$ & $1 D$ & $5 / 9$ \\
3 & $2 P$ & $1 D$ & $5 / 18$ & 5 & $2 P$ & $1 S$ & $1 / 9$ \\
3 & $2 P$ & ${ }^{2} S$ & $2 / 9$ & 6 & $1 S$ & $2 P$ & 2 \\
\hline \hline
\end{tabular}

states but recombination to all excited states of the final system has to be considered.

\section{Calculations for $\mathbf{H}$}

The photoionization cross sections and recombination coefficients may be calculated exactly. The problem of the statistical equilibrium of $\mathrm{H}$ in nebulae has been treated in detail by Baker and Menzel, ${ }^{42}$ the only essential approximation for optically thin nebulae being the assumption that, for fixed $n$, the populations are equal for all quantum states $n l m$. Some preliminary calculations by Joslet (unpublished) have indicated that without this assumption the calculated Balmer decrements may be significantly different, the biggest change being in the $\mathrm{H}_{\alpha} / \mathrm{H}_{\beta}$ ratio. Further calculations are being made by Burgess at University College, London.

${ }^{42}$ J. G. Baker and D. H. Menzel, Astrophys. J. 88, 52 (1938). 


\section{Photoionization from $2 p^{q}$ Configurations}

Calculations have been made $\S$ for all ions with outer $2 p^{q}$ configurations likely to be of importance in gaseous nebulae. Exchange atomic wave functions have been used for $\| \mathrm{Ne}^{0}, \mathrm{O}^{0}, \mathrm{~N}^{0}, \mathrm{C}^{0}, \mathrm{Na}^{+}, \mathrm{Ne}^{+}, \mathrm{O}^{+}$, $\mathrm{N}^{+}, \mathrm{Mg}^{+2}, \mathrm{Ne}^{+2}, \mathrm{O}^{+2}, \mathrm{Ne}^{+3}, \mathrm{O}^{+3}$, and $\mathrm{Ne}^{+4}$. Some use has also been made of approximate analytic wave functions and of a general formula obtained by Burgess and Seaton. ${ }^{43}$ Continuum wave functions calculated with allowance for potential and exchange distortion have been used for ionization of the neutral atoms and singly ionized positive ions. For the higher stages of ionization, distortion effects were found to be of little importance. The cross sections may be expressed as

$$
\begin{array}{rl}
a_{\nu}=10^{-18} & C\left(p^{q} S L \rightarrow p^{q-1} S^{\prime} L^{\prime}\right) \\
& \times B\left\{\alpha\left(\frac{\nu}{\nu_{0}}\right)^{-s}+(1-\alpha)\left(\frac{\nu}{\nu_{0}}\right)^{-s-1}\right\} \mathrm{cm}^{2} .
\end{array}
$$

The coefficients $C$ given in Table XI have been cal-

TABLE XII.

\begin{tabular}{llllllll}
\hline Ion & $B$ & $s$ & $\alpha$ & Ion & $B$ & $s$ & $\alpha$ \\
\hline $\mathrm{Ne}^{0}$ & 2.5 & 1 & 4.3 & $\mathrm{Na}^{+}$ & 4.0 & 2 & 4.2 \\
$\mathrm{~F}^{0}$ & 3.7 & 1 & 4.1 & $\mathrm{Ne}^{+}$ & 5.0 & 2 & 3.8 \\
$\mathrm{O}^{0}$ & 5.7 & 1 & 4.0 & $\mathrm{~F}^{+}$ & 6.4 & 2 & 3.1 \\
$\mathrm{~N}^{0}$ & 8.9 & 1 & 3.1 & $\mathrm{O}^{+}$ & 8.1 & 2 & 2.45 \\
$\mathrm{C}^{0}$ & 15.8 & 1 & 1.9 & $\mathrm{~N}^{+}$ & 10.2 & 2 & 2.06 \\
$\mathrm{~B}^{0}$ & 31 & $\ldots$ & $\cdots$ & $\mathrm{C}^{+}$ & 12.9 & 2 & 1.67 \\
& & & & & & & \\
$\mathrm{Mg}^{+2}$ & 2.6 & 2 & 2.65 & $\mathrm{Al}^{+3}$ & 1.9 & 2 & 1 \\
$\mathrm{Na}^{+2}$ & 3.2 & 2 & 2.4 & $\mathrm{Mg}^{+3}$ & 2.3 & 2 & 1 \\
$\mathrm{Ne}^{+2}$ & 3.82 & 2 & 2.07 & $\mathrm{Na}^{+3}$ & 2.7 & 2 & 1 \\
$\mathrm{~F}^{+2}$ & 4.5 & 2 & 1.7 & $\mathrm{Ne}^{+3}$ & 2.95 & 2 & 1.20 \\
$\mathrm{O}^{+2}$ & 5.23 & 2 & 1.30 & $\mathrm{~F}^{+3}$ & 3.1 & 2 & 1 \\
$\mathrm{~N}^{+2}$ & 6.0 & 2 & 0.9 & $\mathrm{O}^{+3}$ & 3.33 & 3 & 1.82 \\
$\mathrm{Si}^{+4}$ & 1.6 & 2.3 & 1 & & & & \\
$\mathrm{Al}^{+4}$ & 1.8 & 2.3 & 1 & & & & \\
$\mathrm{Mg}^{+4}$ & 1.9 & 2.3 & 1 & & & & \\
$\mathrm{Na}^{+4}$ & 2.0 & 2.3 & 1 & & & & \\
$\mathrm{Ne}^{+4}$ & 2.16 & 2.31 & 1 & & & & \\
$\mathrm{~F}^{+4}$ & 2.4 & 2.3 & 1 & & & & \\
\hline
\end{tabular}

culated from first principles and found to be in agreement with values tabulated by $\operatorname{Bates}^{44}\left(C=C_{l-1}\right)$. In the notation of Seaton, ${ }^{2(a)}$

$$
B=1.71 I\left\{\sigma_{l+1}(L) \sigma_{l+1}(V)+\frac{1}{2} \sigma_{l-1}(L) \sigma_{l-1}(V)\right\}
$$

$\S$ For previous calculations, see D. R. Bates and M. J. Seaton, Monthly Notices Roy. Astron. Soc. 109, 698 (1949); M. J. Seaton, Proc. Roy. Soc. (London) A208, 408 (1951); L. H. Aller, Gaseous Nebulae (Chapman and Hall, Ltd., London, 1956), p. 145.

$\|$ I am indebted to Miss C. Froese for the $\mathrm{Ne}^{+4}$ results and to D. R. Hartree for $\mathrm{Ne}^{+3}$.

${ }^{43}$ A. Burgess and M. J. Seaton, Revs. Modern Phys. 30, 992 (1958), following paper.

4 D. R. Bates, Monthly Notices Roy. Astron. Soc. 106, 432

\begin{tabular}{|c|c|c|}
\hline$\lambda(\mathrm{A})$ & Measured & ${ }^{10^{18}} \times a_{\nu} \quad$ Calculated \\
\hline $\begin{array}{l}573 \\
500 \\
400 \\
300\end{array}$ & $\begin{array}{l}4.8 \\
7.2 \\
7.7 \\
5.4\end{array}$ & $\begin{array}{l}5.0 \\
6.2 \\
7.0 \\
6.7\end{array}$ \\
\hline
\end{tabular}
(1946).
TABLE XIII. Photoionization of $\mathrm{Ne}^{0}$.

the product of the dipole length and dipole velocity matrix elements being used. For all the positive ions the $\sigma_{l-1}$ are small compared to the $\sigma_{l+1}$. Values of $B$ not calculated directly may be estimated with some precision using the fact that $I B$ varies little from one ion to another. The frequency is $\nu$, threshold frequency $\nu_{0}$. The quantities $B, s$, and $\alpha$ are given in Table XII, and the calculated results for $\mathrm{Ne}^{0}$ are compared with measurements made by Po Lee and Weissler ${ }^{45}$ in Table XIII. The positive ion results, which are less sensitive to the wave functions than those for neutral atoms, are probably correct to within $\pm 20 \%$ in most cases.

\section{Photoionization from Valence Electron States}

Using the quantum defect method, calculations for photoionization from valence electron states, similar to the Bates and Damgaard ${ }^{46}$ calculations for transition probabilities, have been made by Burgess and Seaton. These are reported in the following paper. ${ }^{43}$

\section{Photoionization of Neutral Atomic $\mathrm{Ca}$}

The cross section for photoionization of $\mathrm{Ca}^{0}$ is required for the interpretation of $\mathrm{Ca}^{0} / \mathrm{Ca}^{+}$abundance ratios deduced from observations of interstellar $\mathrm{CaI}$ and CaII lines. Laboratory measurements by Jutsum ${ }^{47}$ give a threshold cross section in very poor agreement with results obtained using the Hartree-Fock method. ${ }^{48}$ This discrepancy has been explained as a consequence of configuration interaction and an attempt has been made $^{2(\mathrm{~g})}$ to extrapolate the measured cross section to higher energies. Further laboratory work by T. R. Kaiser (private communication) has shown this extrapolated cross section to be incorrect. $\Upsilon$

${ }^{45}$ Po Lee and G. L. Weissler, Proc. Roy. Soc. (London) A220, 71 (1953).

${ }^{46}$ D. R. Bates and A. Damgaard, Phil. Trans. Roy. Soc. London A242, 101 (1949).

47 P. J. Jutsum, Proc. Phys. Soc. (London) A67, 190 (1954).

48 D. R. Bates and H. S. W. Massey, Proc. Roy. Soc. (London) A177, 329 (1941).

ๆ Note added in proof (June 17, 1958).--Improved calculations [Burgess, Monthly Notices Roy. Astron. Soc. (to be published)] give radiative Balmer decrements similar to those obtained by Baker and Menzel. A recent experimental determination of the elastic electron-hydrogen cross section [Brackmann, Fite, and Neynaber, Phys. Rev. (to be published) ] is consistent with the results of Table X. Field [Proc. Inst. Radio Engrs. 46, 240 (1958)] obtains cross sections for (49) in close agreement with our results. 


\section{DISCUSSION}

L. SPITZER, JR., Princeton University, Observatory, Princeton, New Jersey: From a casual glance at the energy-level diagram, one might suspect that the ratio of the upper transition to the lower transition would depend very much on the temperature, perhaps more on the temperature than the pressure. Can you explain how it is that the temperature disappears in these results?

M. J. Seaton, University College, London, England: We have put all the blame on the variations in density. Maybe we could have proceeded in a different way and assumed the density to be uniform and that there were differences in temperature. In point of fact we have made other approximations which may tend to some extent to cancel the approximation that the temperature is constant. We have assumed that the ratio $n\left(\mathrm{O}^{+}\right) / n_{e}$ is constant, which means that we are assuming that the ionization equilibrium remains the same in the dense regions and the less dense regions. Now this is certainly not going to be correct, but at least the approximation of taking the temperature to be constant and of taking this ratio to be constant go in different directions, and they tend to cancel each other in our integrals.

G. MÜNCH, California Institute of Technology, Pasadena, California: I would like to ask if any estimate can be made of the linear scale of the variations in density. How large is the dimension of the region over which the variation amounts to a factor of 15 ?

M. J. SEATON : All I have been speaking about depends entirely on the spectrum and, of course, from this one cannot deduce anything about the scale, so I suggest the question should be addressed to Minkowski.

R. MINKowSKI, Mount Wilson and Palomar Observatories, Pasadena, California: NGC 7027 is a very small object. It is of the order of $10 \mathrm{sec}$ of arc, and on the best 200-in. photographs which we have, one can just see that it is not the structureless cloud it looked to be on the older pictures, but that there are condensations. But I do not know what the smallest condensations are and whether the larger ones which one sees are not groups of smaller ones.

G. MÜNCH : I wonder if Wilson can tell what is the velocity width of the lines. Is it purely thermal or is there some turbulent motion?

O. C. WILson, Mount Wilson and Palomar Observatories, Pasadena, California: In this nebula the lines are double. This is standard in practically all lines of planetaries. The nebula is expanding in the usual fashion. The lines themselves are not particularly wide and my impression is that the turbulence is small. I have never measured the individual components, but there is nothing exceptionally large about them.

H. ZANSTRA, Sterrenkundig Instituut, Amsterdam, Netherlands: It would be desirable to look for observational criteria to settle whether actually condensations are to be made responsible for the electron concentrations from relative intensities of forbidden lines coming out larger than those from surface brightness (or the absolute $\mathrm{Ba}_{c}$ intensity).

R. MINKOWSKI : I think it is a quite likely thing that this difference in concentrations is really due to condensations, and I would again refer to the observers that it would be very desirable to get means of confirming this highly interesting interpretation.

L. SPITZER, JR.: If Zanstra's mechanism were correct, am I right in assuming that you would then have to.blame the observed differences more on the temperature than on the densities? In such a case, the pressure would be constant throughout the nebula; the regions of high temperature would be regions of low density and vice versa.

M. J. SEaton : I think we would still have the main conclusion that it would be necessary to introduce density differences, but the numerical results might be different. What, eventually, we shall have to do from the theoretical side, is to make a quantitative study of the ionization equilibrium in nebulae. For this study, our biggest handicap has been lack of atomic cross sections; I will report on the status of this material on Thursday. Given the results of such a study, it should be possible to investigate the Zanstra mechanism in much more detail. Theoretical models could be constructed with different conditions of temperature and density in different regions and a model could then be chosen consistent with the observations.

M. MINNAERT : The method which has been used by Seaton seems perfectly general. Is it possible to apply it to the few planetaries where the conditions of observation are much more favorable and where condensations are really observable and visible?

M. J. SEATON: The nebulae that look the most irregular are the ones for which we obtain the most striking condensation effects. An observational difficulty is that when you look at the main body of a nebula, you see superimposed in the line of sight many regions 
of different density and different emissivity, and then it is difficult to see the structure. It is only when you look at the rim of the nebula, where the over-all amount of matter is thinning out, that the structure can be seen most clearly.

H. ZANSTRA : One remark occurs to me for finding an observational criterion for condensations in which the forbidden lines would occur. If one assumes pressure equality (although this may not be true because of magnetic fields, etc.), one should not forget that the electron temperature can be determined from relative intensities of forbidden lines. This would apply to the region where the forbidden lines are formed. Now, if we had another electron temperature determination, say, from hydrogen Balmer continuum or something, and we got an electron temperature a good deal higher, I think one would have a measure of condensation directly (under the assumed pressure equality).

M. J. Seaton: I think there are some exciting possibilities in what Zanstra suggests. 